\title{
Construction of Chaotic Dynamical System
}

\section{Bula ${ }^{1}$ and I. Rumbeniece ${ }^{2}$}

\author{
${ }^{1}$ Institute of Mathematics and Computer Science of University of Latvia \\ ${ }^{1}$ Rainga bulv. 29, Rīga, LV-1048, Latvia \\ E-mail(corresp.): ibula@lanet. Iv \\ ${ }^{1,2}$ University of Latvia \\ ${ }^{1,2}$ Zellyu 8, Rīga, LV-1002, Latvia \\ E-mail: navita@one.lv
}

Received September 2, 2009; revised December 15, 2009; published online February 15, 2010

\begin{abstract}
The first-order difference equation $x_{n+1}=f\left(x_{n}\right), n=0,1, \ldots$, where $f: \mathbf{R} \rightarrow \mathbf{R}$, is referred as an one-dimensional discrete dynamical system. If function $f$ is a chaotic mapping, then we talk about chaotic dynamical system. Models with chaotic mappings are not predictable in long-term. In this paper we consider family of chaotic mappings in symbol space $\Sigma_{2}$. We use the idea of topological semi-conjugacy and so we can construct a family of mappings in the unit segment such that it is chaotic.
\end{abstract}

Keywords: chaotic mapping, infinite symbol space, increasing mapping, topological semi-conjugacy, binary expansion.

AMS Subject Classification: 37B10; 37B05; 37C15.

\section{Introduction}

The theory of discrete dynamical systems and difference equations developed greatly during the last twenty five years of the twentieth century, following the T.Y. Li and J.A. Yorke paper [9] in 1975. In 1986 R. Devaney published [4], the first book on the subject. Discrete dynamical systems are applied in many scientific disciplines (for example, in biology, see article [11]).

Starting from a point $x_{0}$, one may generate the sequence

$$
x_{0}, f\left(x_{0}\right), f\left(f\left(x_{0}\right)\right)=f^{2}\left(x_{0}\right), \ldots, f\left(f^{n}\left(x_{0}\right)\right)=f^{n}\left(x_{0}\right), \ldots
$$

This iterative procedure is an example of a discrete dynamical system. If we let $x(n)=f^{n}\left(x_{0}\right)$, then we obtain the first-order difference equation

$$
x_{n+1}=f\left(x_{n}\right), \quad n=0,1,2, \ldots
$$

We may conclude that difference equations and discrete dynamical systems represent two sides of the same coin. Difference equations represent analytic 
theory of the subject but discrete dynamical systems represent its geometrical and topological aspects. If we consider the logistic difference equation

$$
x_{n+1}=h_{4}\left(x_{n}\right)=4 x_{n}\left(1-x_{n}\right)
$$

or the doubling equation

$$
x_{n+1}=D\left(x_{n}\right)=2 x_{n}(\bmod 1),
$$

then we know that mappings $h_{4}$ and $D$ are chaotic in $[0 ; 1]$. In 1994 H.O. Peitgen, H. Juergen, D. Saupe [12] have shown that doubling mapping $D$ is topological semi-conjugate with the shift map in one-sided infinite sequences space $\Sigma_{2}$. The shift map is chaotic too. We use this idea of topological semiconjugacy and show a possibility of construction of chaotic discrete dynamical systems. Models with chaotic mappings are not predictable in long-term.

\section{Basic Definitions}

Definition 1. ([7, 13]). The set of all infinite sequences of symbols 0 and 1 is called the symbol space of 0 and 1 and is denoted by $\Sigma_{2}$, i.e.,

$$
\Sigma_{2}=\left\{s_{0} s_{1} s_{2} \ldots \mid s_{i}=0 \text { or } s_{i}=1, i=0,1,2, \ldots\right\} .
$$

We introduce a metric structure on $\Sigma_{2}$ by

$$
\forall s=s_{0} s_{1} s_{2} \ldots, t=t_{0} t_{1} t_{2} \ldots \in \Sigma_{2}: \quad d(s, t)=\sum_{i=0}^{+\infty} \frac{\left|s_{i}-t_{i}\right|}{2^{i}} .
$$

The space $\left(\Sigma_{2}, d\right)$ has many specific and interesting properties (see, $\left.[7,10,14]\right)$. Let $(X, \rho)$ be metric space.

Definition 2. (Devaney, [4]) The function $f: X \rightarrow X$ is chaotic if a) the periodic points of $f$ are dense in $X, \mathrm{~b}) f$ is topologically transitive, c) $f$ exhibits sensitive dependence on initial conditions.

Devaney's definition is not the unique classification of a chaotic map. For example, another definition can be found in Robinson [13]. Also mappings with only one property, i.e. sensitive dependence on initial conditions, frequently are considered as chaotic (see, Gulick [5]).

Definition 3. $([4,6,7,13])$. The function $f: X \rightarrow X$ is topologically transitive on $X$ if

$$
\forall x, y \in X \quad \forall \varepsilon>0 \quad \exists z \in X \quad \exists n \in \mathbf{N}: \quad \rho(x, z)<\varepsilon \& \rho\left(f^{n}(z), y\right)<\varepsilon .
$$

Definition 4. ([4,6, 7, 13]). The function $f: X \rightarrow X$ exhibits sensitive dependence on initial conditions if

$\exists \delta>0 \quad \forall x \in X \quad \forall \varepsilon>0 \quad \exists y \in X \quad \exists n \in \mathbf{N} \quad \rho(x, y)<\varepsilon \& \rho\left(f^{n}(x), f^{n}(y)\right)>\delta$. 
Definition 5. ([4,6,7,13]). Let $A, B \subseteq X$ and $A \subseteq B$. Then $A$ is dense in $B$ if for each point $x \in B$ and each $\varepsilon>0$, there exists $y \in A$ such that $d(x, y)<\varepsilon$.

The shift map

$$
\sigma: \Sigma \rightarrow \Sigma \forall s=s_{0} s_{1} s_{2} \ldots \in \Sigma_{2}: \quad \sigma(s)=s_{1} s_{2} \ldots
$$

is a well known example of a chaotic map (see, $[7,10,13])$. But it is not unique chaotic map in space $\left(\Sigma_{2}, d\right)$.

Definition 6. ([3]) The $\alpha_{m}$-mapping $(m=2,3, \ldots) \alpha_{m}: \Sigma_{2} \rightarrow \Sigma_{2}$ is defined by

$$
\alpha_{m}\left(s_{0} s_{1} s_{2} \ldots\right)=s_{1} s_{2} \ldots s_{m-1} s_{m+1} s_{m+2} \ldots
$$

This mapping is not the $k$ th iteration of the shift map, the $\alpha$-mapping "forgets" two symbols of the sequence in every iteration.

It is possible to prove that the every $\alpha_{m}$-mapping $(m \geq 2)$ is continuous, the set of periodic points of the $\alpha_{m}$-mapping is dense in $\Sigma_{2}$ and the $\alpha_{m}$-mapping is topologically transitive on $\Sigma_{2}$ too. It follows from [1] that the $\alpha_{m}$-mapping is chaotic mapping. If we observe that every $\alpha_{m}$-mapping $(m \geq 2)$ is increasing mapping, then this gives a much shorter proof of the fact that $\alpha_{m}$-mapping is chaotic.

\section{Increasing Mappings}

Let $A$ be a finite alphabet, i.e., a finite nonempty set $\left\{a_{0}, a_{1}, a_{2}, \ldots, a_{n}\right\}$ and the elements are called symbols. We assume that $A$ contains at least two symbols. We consider infinite sequences of symbols over a finite set $A$. One-sided infinite sequence over $A$ is any total map $\omega: \mathbf{N} \rightarrow A$.

The set $A^{\omega}$ contains all infinite sequences. Let $f_{\omega}: A^{\omega} \rightarrow A^{\omega}$ and $\exists f: \mathbf{N} \rightarrow$ $\mathbf{N}$ such that

$$
f_{\omega}(x)=x_{f(0)} x_{f(1)} x_{f(2)} \ldots x_{f(i)} \ldots, \quad i \in \mathbf{N}, x \in A^{\omega} .
$$

In this case the function $f$ is called the generator function of mapping $f_{\omega}$.

Definition 7. ([3]) A function $f: \mathbf{N} \rightarrow \mathbf{N}$ is called positively increasing function if

$$
0<f(0) \text { and } \forall i, j: \quad i<j \Rightarrow f(i)<f(j) .
$$

Definition 8. ([3]) The mapping $f_{\omega}: A^{\omega} \rightarrow A^{\omega}$ is called increasing mapping if its generator function $f: \mathbf{N} \rightarrow \mathbf{N}$ is positively increasing.

The shift map

$$
\sigma: \Sigma \rightarrow \Sigma \quad \forall s=s_{0} s_{1} s_{2} \ldots \in \Sigma_{2}: \sigma(s)=s_{1} s_{2} \ldots
$$

is positively increasing mapping because its generator function $f: \mathbf{N} \rightarrow \mathbf{N}$ is positively increasing:

$$
f(x)=x+1, \quad x=0,1,2, \ldots
$$


Theorem 1. ([3]) The increasing mapping $f_{\omega}: A^{\omega} \rightarrow A^{\omega}$ is chaotic in the set $A^{\omega}$.

In our case $A^{\omega}=\Sigma_{2}$ and $\alpha_{m}$-mapping is increasing mapping because its generator function $f: \mathbf{N} \rightarrow \mathbf{N}$ is positively increasing:

$$
f(x)= \begin{cases}x+1, & x=0,1,2, \ldots, m-2, \\ x+2, & x=m-1, m, m+1, \ldots\end{cases}
$$

Corollary 1. The increasing mapping $f_{\Sigma}: \Sigma_{2} \rightarrow \Sigma_{2}$ is chaotic in the symbol space $\Sigma_{2}$.

Corollary 2. The $\alpha_{m}$-mapping is chaotic in the symbol space $\Sigma_{2}, m=2,3, \ldots$.

But on the other hand we have the following theorem.

Theorem 2. ([3]) If generator function $f$ of mapping $f_{\omega}: A^{\omega} \rightarrow A^{\omega}$ is such that $f(0)=0$, then the generated mapping $f_{\omega}$ is not topologically transitive in the set $A^{\omega}$, i.e., it is not chaotic in the set $A^{\omega}$.

In other words chaotic generated mapping always "forgets" the first symbol of the sequence.

\section{Topological Semi-Conjugacy}

Definition 9. ([13]) Let $f: A \rightarrow A$ and $g: B \rightarrow B$ be functions. A map $h: A \rightarrow B$ is called a topological semi-conjugacy from $f$ to $g$ provided 1$) h$ is continuous, 2) $h$ is onto, and 3) $h \circ f=g \circ h$. The map $h$ is called a topological conjugacy if it is homeomorphism and $h \circ f=g \circ h$.

One essential result for our purpose is the following theorem.

Theorem 3. ([12]) Let $A$ and $B$ be subsets of the metric spaces, $f: A \rightarrow A$, $g: B \rightarrow B$, and $\tau: A \rightarrow B$ be a topological semi-conjugacy of $f$ to $g$. If $f$ is chaotic on $A$, then $g$ is topologically transitive on $B$ and has dense set of periodic points in $B$. If $\tau: A \rightarrow B$ be a topological conjugacy of $f$ and $g$, then $f$ is chaotic on $A$ if and only if $g$ is chaotic on $B$.

In 1994 Peitgen, Juergen, Saupe [12] had shown that for chaotic shift map the corresponding chaotic mapping in a unit segment is

$$
S(x)= \begin{cases}2 x \bmod 1, & x \in[0,1[, \\ 1, & x=1 .\end{cases}
$$

This result suggests to find for chaotic increasing mapping (in $\Sigma_{2}$ ) corresponding chaotic mapping in unit segment. 


\section{Construction of Chaotic Mappings in Unit Segment}

We consider binary expansion of numbers from segment $[0,1]$. It is possible to write every number $x$ from $[0,1]$ in form $x=a_{0} a_{1} a_{2} \ldots$, where $a_{k} \in\{0,1\}$ and $x=a_{0} 2^{-1}+a_{1} 2^{-2}+a_{2} 2^{-3}+\ldots$ For example, $\frac{1}{2}=1000 \ldots$ or $\frac{1}{7}=\overline{001} \ldots$ (infinite sequence which periodically repeats after some fixed length will be denoted by the finite length sequence with an over line).

The number $\frac{1}{2}$ has two binary expansions $1 \overline{0}$ and $0 \overline{1}$. We assume that we consider only first variant of binary expansion. Therefore we consider the set $I=\Sigma_{2} \backslash J$, where

$$
J=\left\{s_{0} s_{1} s_{2} \ldots \in \Sigma_{2} \mid \exists N \geq 0 \forall i \geq N s_{i}=1\right\} .
$$

Then we has a second problem with number 1 , its binary expansion $\overline{1} \notin I$. But $\alpha_{m}(\overline{1})=\overline{1}$ and this point is fixed point for every mapping $\alpha_{m}, m=2,3, \ldots$ and all iterations are same. The sequence $\overline{1}$ is fixed point for every increasing mapping too: $f_{\omega}(\overline{1})=\overline{1}$.

The mapping $\tau: \Sigma_{2} \rightarrow[0,1[$ defined by equality

$$
\forall s=s_{0} s_{1} s_{2} \ldots \in \Sigma_{2} \quad \tau(s)=s_{0} 2^{-1}+s_{1} 2^{-2}+s_{2} 2^{-3}+\ldots
$$

is onto, continuous (see, for example, $[8,12]$ ) but it is not one-to-one. The mapping $\tau: I \rightarrow[0,1[$ is onto, continuous and one-to-one.

Here are more possibilities how the number from segment $[0,1[$ transforms to binary expansion. We use the method from [12]:

$$
\begin{aligned}
& x \in\left[0,1\left[, \quad \tau^{-1}(x)=s_{0} s_{1} s_{2} \ldots, \text { where } s_{i}= \begin{cases}0, & z(x)_{i}<\frac{1}{2}, \\
1, & z(x)_{i} \geq \frac{1}{2}\end{cases} \right.\right. \\
& z(x)_{0}=x, z(x)_{i}=2 z(x)_{i-1} \bmod 1, i=1,2, \ldots
\end{aligned}
$$

If we consider $\tau: I \rightarrow\left[0,1\left[\right.\right.$, then the inverse map $\tau^{-1}$ is not continuous. For example, the sequence $x_{n}=\frac{1}{2}-\frac{1}{2^{n}}, n=1,2, \ldots$, converges to $\frac{1}{2}$ but the sequence $\tau^{-1}\left(x_{n}\right), n=1,2, \ldots$, converges to $0 \overline{1} \notin I$. Therefore $\tau: \Sigma_{2} \rightarrow[0,1[$ and $\tau: I \rightarrow[0,1$ [ are not homeomorphisms and not topological conjugacy.

We have shown that $\alpha_{m}$-mapping is chaotic in symbol space $\Sigma_{2}$. Notice that $\alpha_{m}: J \rightarrow J$ and $\alpha_{m}: I \rightarrow I$. The $\alpha_{m}$-mapping is increasing mapping in $I$ too. It follows that $\alpha_{m}$-mapping is chaotic in subset $I \subset \Sigma_{2}$.

We assume that for the $\alpha_{m}$-mapping exists corresponding to chaotic mapping in segment $[0,1]$. We find for $\alpha_{m}$-mapping corresponding mapping $E_{m}$ in segment $[0,1]$. For this, we make the following numerical experiment: first, we write number $x$ from segment [0,1[ in its binary expansion $s \in I$, second, we consider $\alpha_{m}(s)$, third, we write $\alpha_{m}(s)$ in its decimal expansion $E_{m}(x)$ and make a graph. Finally for $m=2$ we find (see Figure 1)

$$
E_{2}(x)=\left\{\begin{array}{llll}
4 x, & 0 \leq x<\frac{1}{8}, & 4 x-\frac{1}{2}, & \frac{1}{8} \leq x<\frac{3}{8} \\
4 x-1, & \frac{3}{8} \leq x<\frac{4}{8}, & 4 x-2, & \frac{4}{8} \leq x<\frac{5}{8} \\
4 x-\frac{5}{2}, & \frac{5}{8} \leq x<\frac{7}{8}, & 4 x-3, & \frac{7}{8} \leq x<1
\end{array}\right.
$$




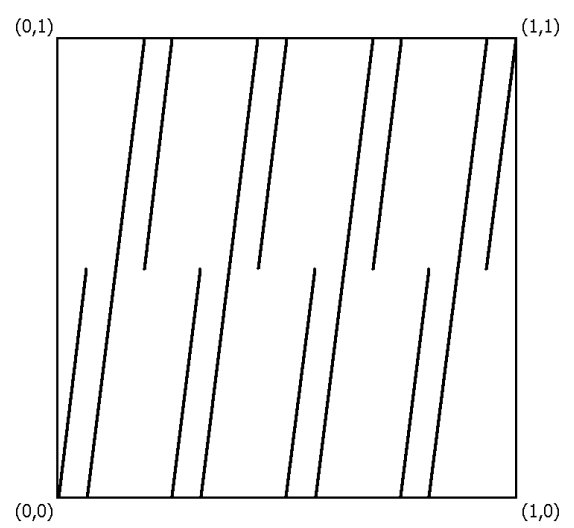

Figure 1. The graph of $E_{2}$.

Similarly we can find $E_{3}, E_{4}, E_{5}, \ldots$ and finally we give formula for $E_{m}$, $m \geq 2$, in general case (see [2]):

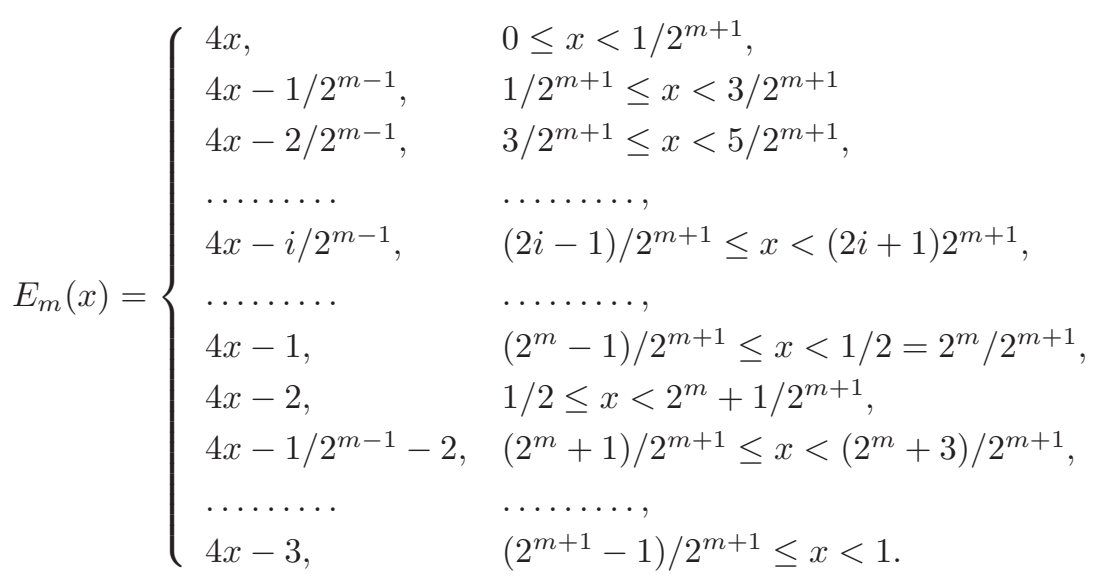

We can prove equality $\tau \circ \alpha_{m}=E_{m} \circ \tau$, i.e., $\tau: I \rightarrow[0,1[$ is topological semi-conjugacy from $\alpha_{m}$ to $E_{m}$. Similarly as for tent map it is possible to prove that $E_{m}:[0 ; 1] \rightarrow[0 ; 1]\left(E_{m}(1)=1\right)$ exhibits sensitive dependence on initial conditions.

Theorem 4. ([2]) Let $E_{m}(1)=1$. Then every mapping $E_{m}:[0,1] \rightarrow[0,1]$, $m=2,3, \ldots$, is chaotic in $[0,1]$.

Construction of a corresponding chaotic mapping in segment [0, 1] for every increasing mapping $f_{\Sigma}: \Sigma_{2} \rightarrow \Sigma_{2}$ can be done in a similar way. We have developed the computer tool that draws graphs of such mappings in the unit segment. The software is presented in http://www.webtech.lv/projects/math/. In this program we indicate only indices of symbols which are "forgotten" in the sequence. In finish we have the graph of corresponding mapping in 


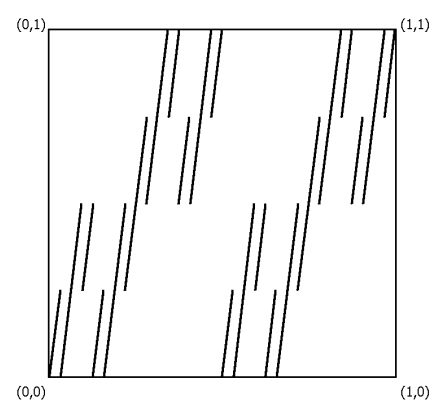

a)

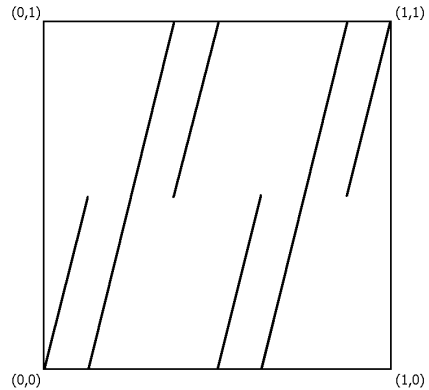

b)

Figure 2. The graph of function in $[0 ; 1]$ that corresponds to: a) increasing mapping that "forgets" first, third and fifth symbols of the sequence, b) increasing mapping that "forgets" first, second and fourth symbols of the sequence.

the unit segment. Similarly, as above we conclude that acquired mapping is chaotic. Two examples are considered. In Figure 2(a) we present a graph that corresponds to increasing mapping that "forgets" first, third and fifth symbols of the sequence and in Figure 2(b) we show a graph that corresponds to increasing mapping that "forgets" first, second and fourth symbols of the sequence.

\section{Conclusions}

We have considered the class of chaotic mappings in the space of infinite sequences. In particular, we have considered increasing mappings in the space of infinite sequences of two symbols (the space $\Sigma_{2}$ ). In this case we have developed a method of construction of corresponding mappings in unit segment. These mappings are chaotic, therefore models with such mappings are not predictable in long-term.

\section{References}

[1] J. Banks, J. Brooks, G. Cairns, G. Davis and P. Stacey. On Devaney's definition of chaos. Amer. Math. Monthly, 99:29-39, 1992. Doi:10.2307/2324899.

[2] I. Bula. Topological semi-conjugacy and chaotic mappings. In Proc. of the 6th EUROMECH Nonlinear Dynamics Conference, Saint Petersburg, Russia, 2008, 2008. Available from Internet: http://lib.physcon.ru/?item=1603.

[3] I. Bula, J. Buls and I. Rumbeniece. Why can we detect the chaos? Journal of Vibroengineering, 10(4):468-474, 2008.

[4] R. Devaney. An introduction to chaotic dynamical systems. Benjaminn Cummings: Menlo Park, CA, 1986.

[5] D.Gulick. Encounters with chaos. McGraw-Hill, Inc., 1992.

[6] S.N. Elaydi. Discrete Chaos: with applications in science and engineering. Chapman\&Hall/CRC (Taylor\&Francis Group), 2008. 
[7] R.A. Holmgren. A first course in discrete dynamical systems. Springer-Verlag, 1996.

[8] L.D. Kudrjavcev. Kurs matematiceskogo analiza. Vissaja skola, Moskva, Tom I, 1988. (in Russian)

[9] T.Y. Li and J.A. Yorke. Period three implies chaos. Amer. Math. Monthly, 82:985-992, 1975. Doi:10.2307/2318254.

[10] D. Lind and B. Marcus. An introduction to symbolic dynamics and coding. Cambridge University Press, 1995.

[11] R.M. May. Simple mathematical models with very complicated dynamics. $\mathrm{Na}$ ture, 261:459-467, 1976. Doi:10.1038/261459a0.

[12] H.O. Peitgen, H. Juergen and D.Saupe. CHAOS. Bausteine der Ordnung. KlettCotta=Springer-Verlag, 1994. (in German)

[13] C. Robinson. Dynamical systems. Stability, symbolic dynamics, and chaos. CRS Press, 1995.

[14] S. Wiggins. Global bifurcations and chaos. Analytical methods. Springer-Verlag, 1988. 